\title{
Recycling von Multilayerfolien
}

\author{
Gerald Koinig · Bettina Rutrecht · Daniel Vollprecht
}

Angenommen: 19. Oktober 2021 / Online publiziert: 15. November 2021

(C) Der/die Autor(en) 2021

Zusammenfassung Die Verbreitung von Mehrschichtfolien im Bereich Kunststoffverpackungen bringt viele Vorteile für Konsumenten, Vertrieb und Umwelt, stellt aber die Abfallwirtschaft vor neue Herausforderungen. Der Materialverbund aus dünnsten unterschiedlichen Plastikwerkstoffen ist bislang mangels passender Technologien im Industriemaßstab online nur schwer vom restlichen Kunststoffverpackungsstrom unterscheidbar. Der Mix an unerwünschten Materialien führt zu Kompatibilitätsproblemen der Kunststoffe untereinander im Recyclingprodukt und in weiterer Folge zu einer Qualitätsminderung der Sekundärrohstoffe. In Projekten, wie etwa dem Multilayer-Detection-Projekt, wird bereits nach Lösungen dazu gesucht. Ziel ist die bestehende NIR-Sortiertechnik in bestehenden Kunststoffrecyclinganlagen dermaßen zu adaptieren, dass die Identifikation und Ausschleusung der Multilayerfolien mit möglichst geringem Aufwand nachgerüstet werden kann. Mit der Entwicklung der neuen Recyclingmethode können sich die vorhandenen Vorteile der Folienverpackungen bestmöglich und umweltfreundlich entfalten und eine abfallwirtschaftliche Herausforderung durch einen wertvollen Beitrag zur Kreislaufwirtschaft gemeistert werden.

Schlüsselwörter Mehrschichtfolien Kunststoff • Recycling ·

Leichtverpackung .

Nahinfrarotsortierung .

Nahinfrarotspektroskopie .

Kunststoffverpackung .

Kunststofffolien .



B. Rutrecht, BSc

assoz.Prof. Dipl.-Min. Dr.rer.nat. D. Vollprecht

Lehrstuhl für

Abfallverwertungstechnik und

Abfallwirtschaft, Montanuniversität

Leoben, Franz Josef

Straße 18, 8700 Leoben, Österreich

gerald.koinig@unileoben.ac.at
Umweltauswirkungen • Vorteile . Potential · Flexible Verpackungen

\section{Recycling of multilayer films}

Abstract The spread of multilayer films in the field of plastic packaging brings many advantages for consumers, distribution, and the environment but poses new challenges for waste management. The material composite of the thinnest different plastic materials is so far hardly distinguishable from the rest of the plastic packaging stream due to a lack of suitable technologies on an industrial scale. The mix of undesirable materials leads to compatibility problems among the plastics in the recycling product and subsequently to a reduction in the quality of the secondary raw materials. Projects like "The Multilayer Detection project" are currently looking for solutions. The aim is to adapt the existing NIR sorting technology in existing plastics recycling plants in such a way that the identification and rejection of multilayer films can be retrofitted straightforwardly. With the development of the new recycling method, the existing advantages of lightweight foil packaging can be developed in the best possible and environmentally friendly way. A waste management challenge can be mastered and turned into a valuable contribution to the circular economy.

Keywords Multilayer films · Plastic . Recycling · Light weight packaging · Near-infrared sorting $\cdot$ Near infrared spectroscopy $\cdot$ Post-consumer plastic packaging · Plastic films .

Environmental impact - Advantages . Flexible plastic packaging

\section{Einleitung}

Zunehmender Wohlstand, längere Transportwege und steigendes Bewusstsein für die Notwendigkeit längerer Haltbarkeit zur Vermeidung von Lebensmittelabfällen führen zu einem Anstieg an Leichtverpackungen in den Abfallströmen Österreichs.
Der Spagat zwischen der Erfüllung mannigfaltiger Anforderungen wie Gasundurchlässigkeit, UV-Schutz, mechanischer Schutz, Bedruckbarkeit und lange Frischhaltefähigkeit von Nahrungsmitteln bei gleichzeitig minimalem Gewicht zur Einsparung von Treibhausgasen beim Produkttransport verlangen nach Multilayerfolien, einem High-Tech-Produkt, welches all diesen Anforderungen gerecht werden kann.

\section{Ein unterschätztes High-Tech- Produkt}

Diese High-Tech-Produkte der Kunststoffindustrie vereinen Eigenschaften in sich, wie sie ohne den Einsatz hochentwickelter Kompositwerkstoffe undenkbar wären.

Die Verbindung von Polyethylen (PE) mit Polypropylen (PP) und Polyethylenterephthalat (PET), Ethylenvinylalkohol (EVOH) und einem Haftvermittler bestehend aus mit Maleinsäureanhydrid modifziertem PE zur Erreichung einer leichten, stapelbaren Verpackung mit herausragenden Licht-, Gas- und Wasserbarriereeigenschaften bei gleichzeitig guter Strapazier- und Bedruckbarkeit für ein ansprechendes Äußeres, ist nur eines der Beispiele zur Anwendung von Multilayerfolien.

Diesen Erfolg spiegelt auch der steigende Marktanteil wieder, den flexible Verpackungen für sich beanspruchen dürfen.

Im Jahr 2010 entfielen $21 \%$ des europäischen Verpackungsverbrauchs auf flexible Verpackungen, was einem Umsatz von 42,9 Mio. US Dollar oder umgerechnet etwa 36,1 Mio. $€$ entspricht, womit flexible Verpackungen die nach Kartonagen umsatzstärkste Verpackungssparte sind. (Pira 2011) (Abb. 1).

Die Nachfrage der EuropäerInnen nach Kunststoffverpackungen erreichte 2015 47,8 Mio. Tonnen. Davon entfielen etwa $32 \%$ auf die Verpackung von Lebensmitteln, wovon geschätzte 0,8 Mio. Tonnen auf Multilayermaterialien entfielen (PlasticsEurope 2015). 


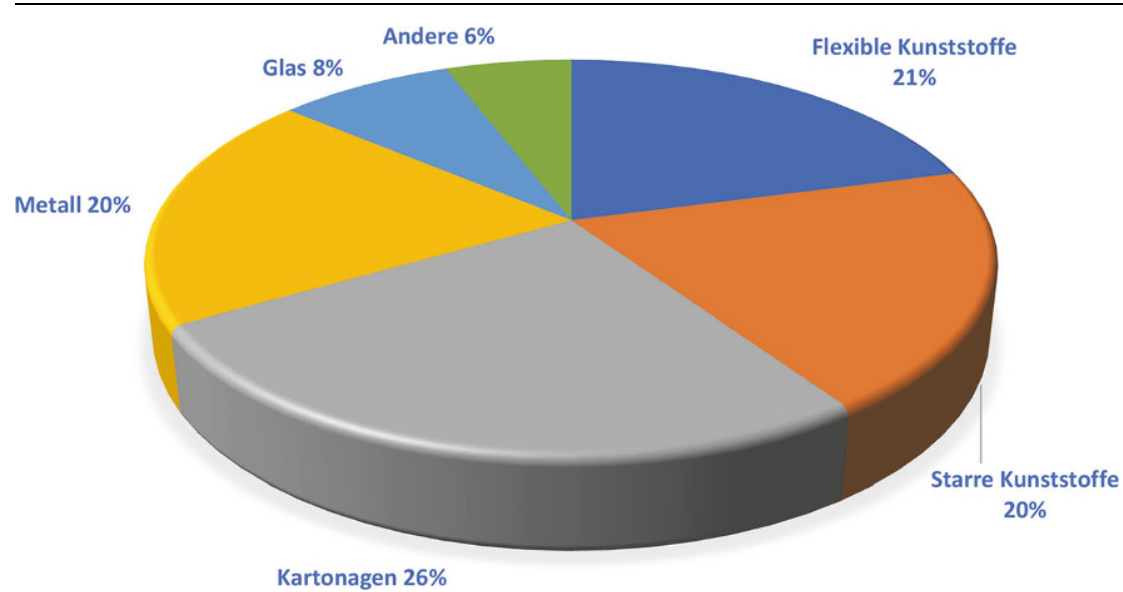

Abb. 1 Verteilung der Verpackungsmaterialien

Während Monolayerfolien für die derzeitigen Anlagen kaum Probleme darstellen, sobald sie in den Prozess eingebracht worden sind, gelten Multilayermaterialien als die größte Herausforderung für die Aufbereitung der 2DFraktion. Beiden Fraktionen gemein ist das Ziel, sie vermehrt zu sammeln, aufzubereiten und die thermische Verwertung dieser Fraktionen zu reduzieren.

\section{Plastik gegen die Wegwerfgesellschaft}

Einfache Verpackung kann die Haltbarkeit von Lebensmitteln stark erhöhen, wie in Tab. 1 aus Daten in einer Studie von „Denkstatt“ (2016) im österreichischen Handel erhoben, hervorgeht. Die Studie untersuchte den Effekt, den korrekte Verpackung auf die Haltbarkeit und den damit verbundenen Lebensmittelabfall haben kann, bei fünf verschiedenen Lebensmitteln. Diese wurden ausgesucht, da sie repräsentativ für einen Großteil der Produktgruppen bei einem typischen Nahversorger sind.

Flexible Verpackungen und besonders Multilayermaterialien ermöglichen die Erfüllung einer ganzen Palette an Anforderungen, den Erhalt wertvoller Ressourcen und die Schonung der Umwelt durch weniger Lebensmittelabfälle.

Diese Funktion vollführen sie durch ihre Flexibilität, wodurch sich die Verpackung an das Produkt anpassen lässt, ohne Luft mitverpacken zu müssen. Die Möglichkeit, mehrere Materialien zu einem Kompositmaterial zusammenzufügen, erlaubt es dem Hersteller, so gut wie jede erdenkliche Aufgabe mit minimalem Materialeinsatz zu erfüllen. Die Formgebungsmöglichkeiten der Kunststoffe erlauben es weiters, nahezu jede gewünschte Form zu erreichen und Features wie Zipper in die Verpackung $\mathrm{zu}$ integrieren, wodurch sich wiederverschließbare Verpackungen verwirklichen lassen.

\section{4 Öffentliche Wahrnehmung von Folienverpackungen}

Nun ist es notwendig, mit einem Vorurteil zu brechen. Wie Umfragen in den USA ergaben, sehen $89 \%$ der Konsumenten Verpackung als überflüssigen Plastikabfall. Weiters werden minimal oder nicht verpackte Lebensmittel als umweltfreundlich und ökologisch betrachtet (Sealed Air 2014). Dies in Betracht der Tatsache, dass etwa $30 \%$ aller Lebensmittel zu Abfall werden (Wohner et al. 2019), führt zu einem höheren Aufkommen an Lebensmittelabfällen als notwendig und damit zu einer immensen Belastung der Umwelt.

Es konnte anhand von Lebenszyklusanalysen gängiger Lebensmittel gezeigt werden, dass die Verpackung nur etwa 5 bis $10 \%$ der gesamten Umweltbelastung ausmacht, die durch das Produkt verursacht wird (Silvenius et al. 2011; Büsser et al. 2007, Büsser und Jungbluth 2009, 2011).

Da die Erfassung des Einflusses von Lebensmittelabfällen in Lebenszyklusanalysen (LCA) von Verpackungsmaterialien aufgrund der unzuverlässigen und raren Daten zum KonsumentInnenverhalten nur schwer quantifizierbar ist, gibt es in dieser Hinsicht nur wenige LCA. Eine dieser wenigen verfügbaren Analysen wurde 2013 von SIlvenius et al. durchgeführt und inkludiert die $\mathrm{CO}_{2}$-Emission verursacht durch die Entsorgung des in der Studie
Tab. 1 Lebensmittelabfallreduzierung durch geeignete Verpackung (Denkstatt 2016)

\begin{tabular}{|l|l|l|}
\hline Produkt & $\begin{array}{l}\text { Lebensmittelabfall } \\
\text { bei Standardver- } \\
\text { packung (in \%) }\end{array}$ & $\begin{array}{l}\text { Lebensmittelabfall } \\
\text { bei verbesserter } \\
\text { Verpackung (in \%) }\end{array}$ \\
\hline Steak & 34 & 18 \\
\hline Käse & 5 & 0,14 \\
\hline Hefe & 11 & 0,8 \\
\hline Kresse & 42 & 3,4 \\
\hline Gurken & $\begin{array}{l}9,4 \text { (ohne Verpa- } \\
\text { ckung) }\end{array}$ & 4,6 \\
\hline
\end{tabular}

betrachteten Lebensmittels: geschnittenes, verpacktes Brot.

Hier konnte gezeigt werden, dass bereits die Entsorgung einer halben Scheibe Brot die Kohlenstoffdioxidemission der Verpackung, inklusive Entsorgung, überstieg (Silvenius et al. 2011).

\section{Overpackaging und Underpackaging}

Gemäß dem Innventia AB-Verpackungsmodell (The Consumer Goods Forum 2011), dargestellt in Abb. 2, ist weiters $\mathrm{zu}$ beachten, dass die Erzeugung von Lebensmittelabfällen durch zu sparsamen Einsatz von Verpackungsmaterial, sogenanntes Underpackaging, gravierendere ökologische Einflüsse nach sich zieht, als die Haltbarkeit des Produktes durch leichtes Overpackaging zu garantieren.

Die richtige Balance zwischen diesen beiden Extremen zu finden ist die Verantwortung der Verpackungsindustrie und dient sowohl der Schonung wertvoller Ressourcen als auch der Verringerung der $\mathrm{CO}_{2}$-Emissionen.

Der positive Effekt, den Folienverpackungen auf den ökologischen Fußabdruck haben, kann noch weiter verstärkt werden, indem ideales Verpackungsdesign verfolgt wird, welches das Produkt weder über- noch unterverpackt. Dies ist mit flexiblen Verpackungen, aufgrund der eingangs erwähnten Vorzüge, leichter $\mathrm{zu}$ erreichen als mit anderen Verpackungsarten.

\section{Potenzial in der Anwendung von flexiblen dünnschichtigen Verpackungen}

Das Potenzial, welches noch in der verstärkten Anwendung von flexiblen dünnschichtigen Verpackungen aus Kunststoffen schlummert, wurde 2014 in einer Studie des Instituts für Energie- und Umweltforschung Heidelberg 


\section{Originalarbeit}



Increasing Packaging Material Weight or Volume $\rightarrow$

Abb. 2 Innventia AB-Verpackungsmodell (Nach The Consumer Goods Forum 2011)

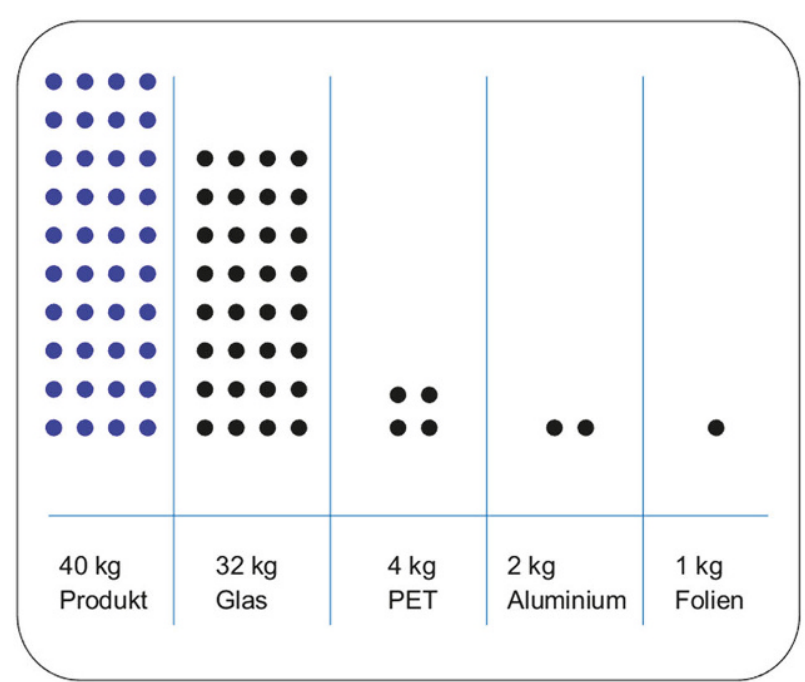

Abb. 3 Vergleich dernotwendigen Verpackungsmenge, um $40 \mathrm{~kg}$ flüssiges Produkt zu verpacken. (Nach FPA2009)

(IFEU) untersucht. Dieser Untersuchung lag die Annahme zugrunde, dass sämtliche Lebensmittelverpackungen in Form von Folien ausgeführt werden. Gleichzeitig wurde als Szenario untersucht, welchen Effekt ein völliger Umstieg von Folien auf Verpackungen aus starren Kunststoffen auf die Umwelt hätte.

Die Ergebnisse waren, dass der Ersatz der 3,79 Mio. Tonnen Folienverpackungen den Einsatz von 20,5 Mio.
Tonnen starrer Kunststoffverpackung notwendig machen würde. Dies macht die Ressourceneffizienz von Folienverpackungen klar deutlich. Außerdem würde ein Umstieg auf starre Verpackungen den $\mathrm{CO}_{2}$-Fußabdruck um 5,6\% erhöhen und den Wasserverbrauch um $5,3 \%$ steigern. Hier wurde außerdem noch die Annahme getroffen, dass $100 \%$ der starren Verpackungen recycelt werden würden. Dieses Szenario stellt also einen Best-Case-Ansatz dar.
Die gegensätzliche Annahme ging davon aus, dass die derzeit etwa 30 Mio. Tonnen starrer Plastikverpackungen durch Folienverpackungen ersetzt werden würden, wobei kein Anteil der Folienverpackungen einer Wiederverwertung, mit Ausnahme der thermischen Verwertung, zugeführt werden würde. Dieses Worst-Case-Szenario ergab, dass die derzeit etwa 30 Mio. Tonnen an starrer Verpackung durch etwa 4,22 Mio. Tonnen Folienverpackung zu ersetzen seien. Diese Verringerung um etwa $77 \%$ der gesamten Verpackungsmasse ergab im Rahmen der LCA eine Reduktion des $\mathrm{CO}_{2}$-Fußabdrucks um etwa $40 \%$ und eine Verringerung des Wasserverbrauchs um etwa $44 \%$ (Ifeu 2014).

\section{Vergleich Glasverpackung und Folienverpackung}

Neben diesen ökologischen Vorteilen wirkt sich die Anwendung flexibler Verpackungen auch auf die Transportkosten aus. Der Transport von Folienverpackungen in Form von Rollen ist im Vergleich zum Transport von Containern, wie PET-Flaschen, Kisten etc. praktikabler und wegen des geringeren Anteils an umpackter Luft kostensparender, wie von der Flexible Packaging Association (2009) bereits dargestellt wurde. 


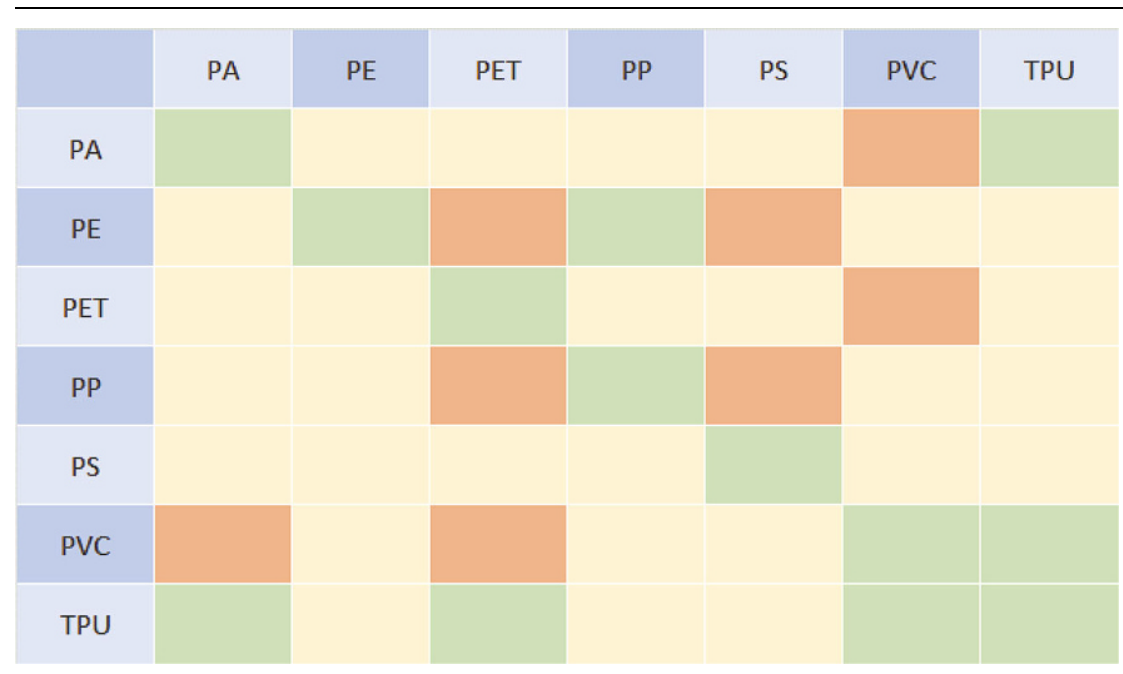

Abb. 4 Kompatibilität der gängigsten Kunststoffe. (PA Polyamid, PE Polyethylen, PET Polyethylenterephthalat, PP Polypropylen, PS Polystyrol, PVC Polyvinylchlorid, TPU Thermoplastisches Polyurethan). (Auszug nach Eco3E 2016)

Weiters wird für die Verpackung des gleichen Produktvolumens weit weniger Verpackungsmasse benötigt, wodurch ein günstigeres Produkt-zuVerpackungs-Verhältnis möglich ist. Dadurch wird beim Transport von Produkten weniger zulässige Gesamtmasse des Transportvehikels, etwa eines Lkw, für die Masse der Verpackungen verwendet, wodurch weniger Lkw notwendig sind, um dieselbe Menge an Produkten zu transportieren. Hierdurch werden sowohl das Verkehrsnetz entlastet als auch $\mathrm{CO}_{2}$-Emissionen vermieden. Dieses Verhältnis ist in Abb. 3 dargestellt.

Untersuchungen wie diese machen den Mehrwert, den Folienverpackung für Industrie, Gesellschaft und Umwelt darstellt, mehr als deutlich. Dahingehend ist es sowohl ökologisch als auch ökonomisch gerechtfertigt, den Einsatz von Folienverpackungen in den kommenden Jahren $\mathrm{zu}$ forcieren.

\section{Umgang mit Folienverpackungen am Ende ihrer Verwendung}

Neben all diesen Vorteilen trägt das 2DVerpackungssegment zur derzeit geringen Recyclingquote von Kunststoffverpackungen bei.

Nach der Änderung der Berechnungsmethode ist die österreichische Recyclingquote von vormals $32 \mathrm{~m} \%$ auf $25 \mathrm{~m} \%$ abgesackt. Bis 2025 soll aber die EU-Vorgabe von $50 \mathrm{~m} \%$ erfüllt werden, und um diese bis dahin $\mathrm{zu}$ erreichen, bedarf es einer großen Gemeinanstrengung. Das Sammelvolu- men an Kunststoffabfällen betrug 2019 $172.029 \mathrm{t}$, wobei davon etwa $26 \mathrm{~m} \%$ auf Kunststofffolien zurückgehen (BMK 2021). Aktuell werden laut ARA Recyclingquoten von $78 \mathrm{~m} \%$ für die Verwertung (hauptsächlich thermisch), $58 \mathrm{~m} \%$ für die Sortierung und weitere $58 \mathrm{~m} \%$ bei der Sammlung erreicht (Scharff 2021). Exemplarisch muss für eine Zielerreichung einer Recyclingquote von $50 \mathrm{~m} \%$ durchwegs eine Recyclingquote von $80 \%$ entlang der Verwertungskette erzielt werden.

Dieser Missstand muss in den nächsten Jahren durch politische Intervention oder durch technische Innovation behoben werden, indem wirtschaftliche Anreize für das verstärkte Recycling dieser Wertstoffe geschaffen werden. $\mathrm{Da}$ das Recycling von flexiblen 2DVerpackungen, insbesondere von Mehrschichtfolien, technischer Innovationen bedarf, ist es notwendig, Forschungsprojekte durchzuführen, die sich mit der Lösung dieser Probleme beschäftigen. Dies ist insofern von besonderem Interesse, da sich Verpackungsfolien mit 45.000 t/a niederschlagen, was einem Massenanteil von etwa $26 \mathrm{~m} \%$ entspricht (Van Eygen 2018). Damit stellen Verpackungsfolien einen beträchtlichen Anteil am derzeit getrennt gesammelten Abfall dar.

Durch die weite Verbreitung von Anlagen zum mechanischen Recycling liegt hier großes Potenzial zur Erhöhung der Recyclingquote, daher muss gerade dieser Bereich weiter erforscht und entwickelt werden. Außerdem stehen chemische Recyclingmethoden derzeit in der Entwicklungsphase (Walker et al. 2020).

Weiters stellt das mechanische Recycling jenen Schritt in der Wertschöpfungskette dar, der durch die gezielte Sortierung von Mehrschichtfolien eine wertschöpfende Rohstoffquelle für die zurzeit in der Entwicklung befindlichen Mehrschicht-Recyclingprozesse schafft.

Ehe jedoch derartige Innovationen greifen können, ist es notwendig, die Sammelquote der 2D-Verpackungsfraktion zu erhöhen.

Grundlage für eine wirtschaftlich attraktive Aufbereitung von flexiblen Verpackungen ist ein Materialstrom von ausreichender Qualität und Quantität. Unter den derzeit gegebenen Voraussetzungen der Kunststoffabfallsammlung sind diese beiden Eigenschaften widersprüchlich und nur sehr schwer vereinbar. Weiters unterliegen die gesammelten flexiblen Verpackungen gerade im Bereich der Konsumentenabfälle stärkeren Schwankungen, als dies bei Industrieabfällen der Fall ist.

Die Kontamination der Folien mit Lebensmittelresten, wie sie bei der Anwendung als Lebensmittelverpackung, der gängigsten Anwendungsform von Folienverpackungen, vorkommt, verursacht in der Aufbereitung Probleme, da die Verschmutzung teilweise 10 bis $20 \%$ des Materialgewichts erreichen kann (Morris 2016). Dies täuscht eine höhere Sammelmasse vor, als tatsächlich für den Rezyklierprozess verwendet werden kann, verkompliziert die Sortierung der Folienfraktion und kontaminiert durch die gemeinsame Sammlung in weiterer Folge ebenfalls jene 2DMaterialien, welche nicht als Lebensmittelverpackung eingesetzt wurden.

Abhilfe können hier separate Sammelsysteme schaffen, welche 2D-Materialien separat von starren Kunststoffverpackungen sammeln, der verstärkte Einsatz von Ballistikseparatoren, oder gar eine Trennung zwischen Lebensmittelverpackungen und anderen Folienverpackungen. Eine weitere Lösung sind neue Technologien, welche sich für die Sortierung von 2D-Materialien eignen, wie etwa Marker, welche durch NIR erkennbar sind. Digitale Wasserzeichen, RFID- oder Barcode-Markierung sind einige der Technologien, welche die Sortierquote von 2D-Materialien erhöhen können (Woidasky et al. 2018).

Daneben ist es notwendig, die Sortierung schwarzer Kunststoffe zu verbessern, nicht nur im Hinblick auf Folienverpackungen, da ein beträchtlicher 


\section{Originalarbeit}

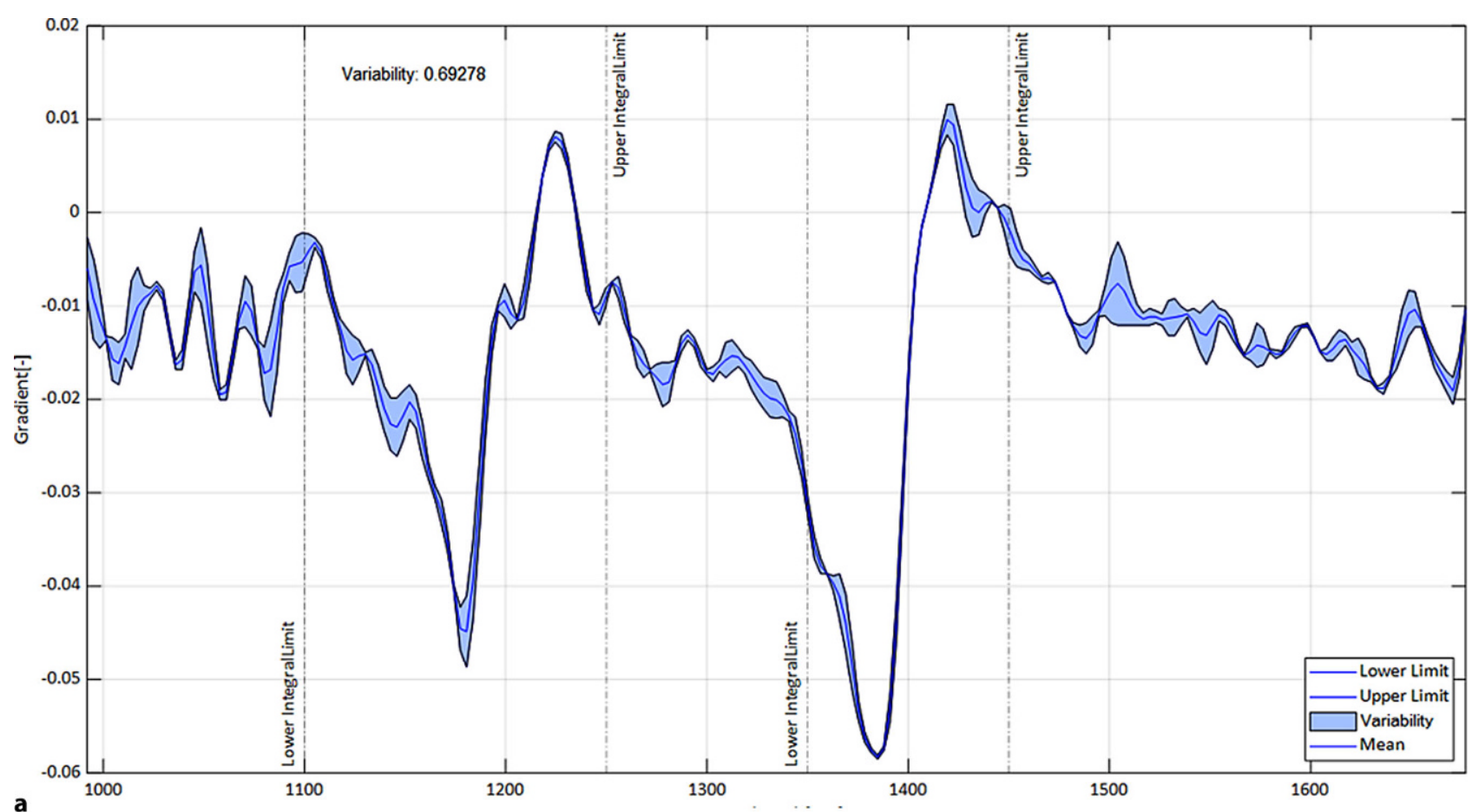

a

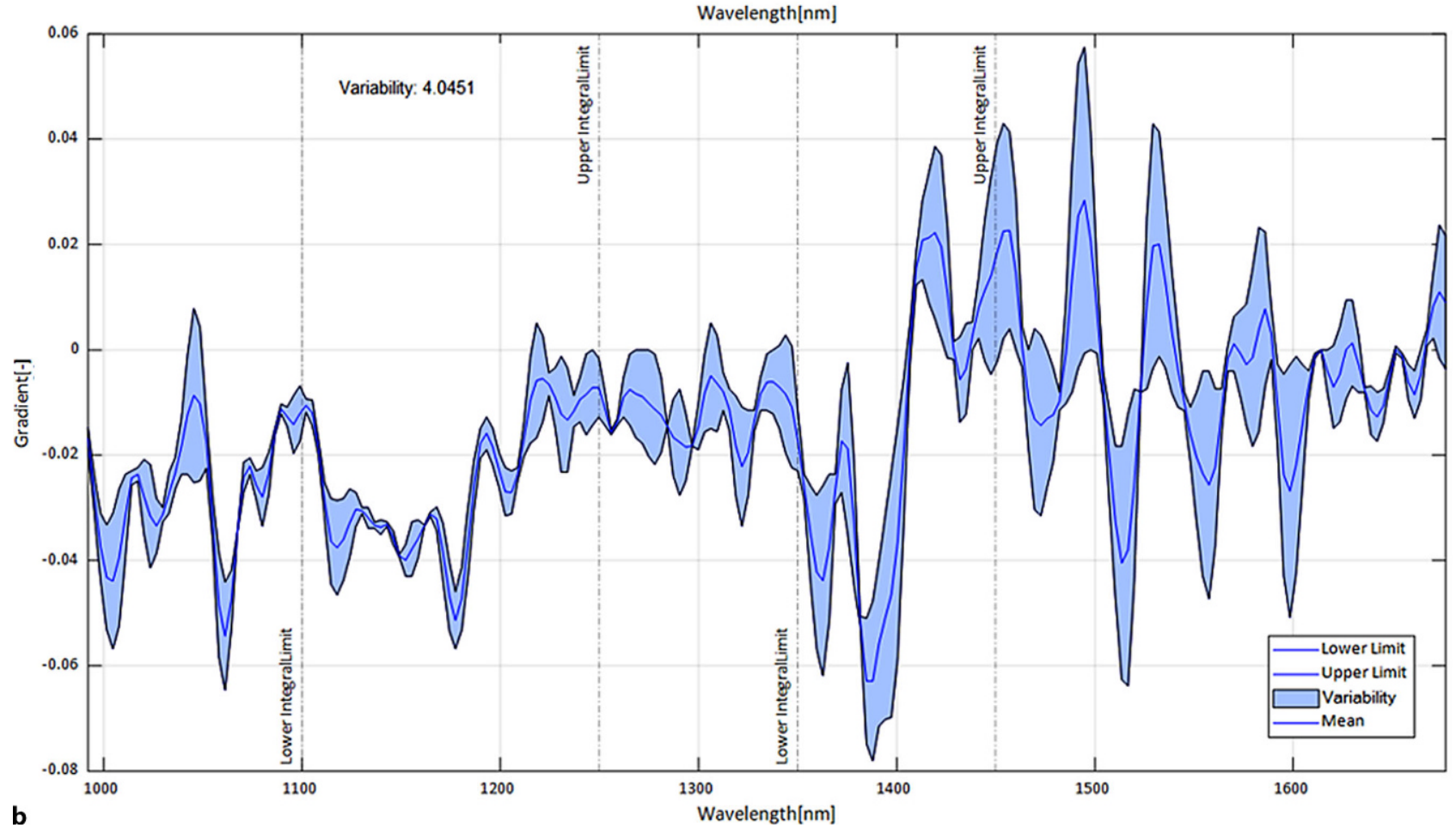

Abb. 5 Vergleich des Spektrumseiner dünnschichten Verpackungsfolie: nachAdaption des Sortieraggregats (a) und ohne Adaption des Sortieraggregats (b)

Anteil aller Kunststoffverpackungen aus schwarzem Kunststoff ist. Eine Lösung stellt hier etwa die Black-Eye-Technologie von Steinert dar (Steinert 2018).
9 Recycling von Multilayermaterialien

Um zu verstehen, warum es wichtig ist, Multilayermaterialien aus dem 2DStrom zu entfernen, muss zuerst auf die
Materialeigenschaften und die Mischbarkeiten der einzelnen Kunststoffsorten eingegangen werden. welche in den Multilayermaterialien am häufigsten zum Einsatz kommen. 
Nicht jedes Material lässt sich ohne Weiteres mit anderen Kunststoffen mischen und verbinden, daher stellt bereits ein geringer Anteil an Störstoffen in einer Reinfraktion eine Kontamination dar, die den Preis dieses Produkts stark verringert oder es gar unbrauchbar werden lässt.

Diese Kompatibilitätsproblematik macht in der Herstellung von Multilayerfolien den Einsatz von Haftvermittlern notwendig und erhöht gleichzeitig die Anzahl an Schichten und Materialien, mit denen es im Rahmen der Aufbereitung umzugehen gilt.

Bereits ein geringer Anteil an inkompatiblen Materialien in der Aufbereitung macht die Herstellung einer homogenen Schmelze unmöglich. Grund dafür sind die unterschiedlichen Materialeigenschaften wie etwa der unterschiedliche Schmelzpunkt von Thermoplasten und Elastomeren. Die Kompatibilität der gängigsten Kunststoffe ist in Abb. 4 dargestellt.

Da hier jeder Hersteller einen eigenen Ansatz verfolgen kann, steigt die Anzahl der möglichen Schichtkombinationen und damit die Menge an unterschiedlichen Multilayerfolien stetig. Eine klare Trennung in einzelne Materialgruppen, wie sie bei Verpackungen aus Monomaterial möglich ist, wird dadurch verhindert. Abhilfe können hier politische Rahmenbedingungen schaffen, die die Menge an möglichen Schichtkombinationen im Namen einer verbesserten Rezyklierbarkeit, Schonung von Ressourcen und der Verbesserung der Kreislaufwirtschaft der Kunststoffe vereinheitlichen.

Die dadurch erleichterte Aussortierung von Mehrschichtfolien erlaubt die Herstellung von Reinfraktionen aus 2DVerpackungsmaterial, welche anschließend einer wertschöpfenden Weiterverarbeitung zu hochwertigen Produkten mit guten mechanischen Eigenschaften zugeführt werden können. Dies ist notwendig, da das Potenzial des Downcyclings, welchem Fraktionen an Folienmaterialien aufgrund der durch Verunreinigungen geringen mechanischen Eigenschaften oft zugeführt werden, ausgereizt ist und die thermische Verwertung keinen Beitrag zur Erhöhung der durch die Europäische Union vorgeschriebenen Recyclingquote liefert (The European Parliament and the Council of the European Union 2018).

Neben soziologischen und politischen Verbesserungsmöglichkeiten zur Sortierung von Mehrschicht- und Mo- nolayerfolien steht die Erforschung von Lösungsansätzen für die erfolgreiche Sortierung der 2D-Fraktion im Mittelpunkt.

Eines der Projekte, welches sich mit dieser Thematik befasst, ist das vom Land Steiermark und dem Zukunftsfonds geförderte Projekt „Multilayer Detection" der Montanuniversität Leoben, in dem zusammen mit dem PCCL untersucht wird, inwiefern die Sortiertechnologie für die Trennung von Mono- und Multilayermaterialien verbessert werden kann.

Ziel des Projekts ist die Erhöhung der Recyclingquote von Verpackungsfolien. In diesem Bereich kommen sowohl Einschicht- als auch Mehrschichtfolien zum Einsatz, wobei letztere mit dem Stand der Technik nicht rezyklierbar sind. Das Projekt zielt nun auf eine neue Sortiertechnik für Verpackungsfolien ab. Erreicht werden soll das durch den Einsatz von neuen Sensorsortiermethoden auf Basis der Nah-InfrarotSpektroskopie, um eine Trennung von rezyklierbaren und nicht-rezyklierbaren Materialien herbeizuführen. Die gezielte Sortierung von Mehrschichtfolien ermöglicht dann eine neue Qualität des Rezyklats mit wesentlich verringerten Fremdstoffanteilen.

Die in diesem Projekt erforschte neue Sortiertechnologie erweitert durch die Erkennung von Mehrschichtfolien die Sortiertiefe von Kunststofffolien und ermöglicht durch den Erhalt von Einschichtfolien eine Integration von bisher nicht berücksichtigten Ressourcen im mechanischen Recycling. Ein erhöhter Anteil der stofflichen Verwertung von Kunststoffabfällen erhöht die Effizienz im mechanischen Recycling und verbessert die Kreislaufwirtschaft von Kunststoffen. Die gezielte Sortierung von Mehrschichtfolien schafft zusätzlich eine wertschöpfende Rohstoffquelle für zurzeit in Entwicklung befindliche Mehrschicht-Recyclingprozesse.

Die Projektergebnisse stellen die Grundlage für die Entwicklung einer industriell nutzbaren Sensortechnologie dar. Eventuell patentierbare Projektergebnisse werden vor einer Veröffentlichung entsprechend evaluiert und geschützt. Diese Veröffentlichungen und das optionale Patent stärken den Ruf Österreichs und der Steiermark und damit den der hier situierten Recyclingunternehmen als technologische und wissenschaftliche Innovatoren.
Erste Ergebnisse zeigen eine verbesserte Spektralgüte durch kostengünstige Adaptionen an einem Stand-derTechnik-Sortiersystem. Die Spektralgüte, welche durch eine herkömmliche kontaktlose Messung von dünnschichtigen Folienverpackungen in Reflektion erreicht werden kann, ist aufgrund der geringen Materialstärke nicht ausreichend für eine Klassifizierung der Materialtype. Effekte wie sinusförmige Spektralüberlagerungen und eine unzureichende Reflektion der Nahinfrarotstrahlung führen $\mathrm{zu}$ unbrauchbaren Spektren für die Datenanalyse. Abb. 5 zeigt den Vergleich eines Spektrums desselben Materials vor und nach der Adaption des Sortieraggregats.

\section{Zusammenfassung}

Flexible Folienverpackung kann einen erheblichen Beitrag zur Schonung von Ressourcen und $\mathrm{zu}$ einer gesteigerten Kreislaufwirtschaft leisten. Insbesondere durch den geringen Ressourceneinsatz, welcher durch flexible Verpackungen notwendig ist, um Lebensmittelabfälle zu minimieren, und das bei idealem Verhältnis von Verpackungsmasse zu Produktmasse.

Dieser Aspekt birgt weiterreichende Vorzüge, so kann durch den verstärkten Einsatz an flexibler Verpackung die Produktlogistik nachhaltiger durch die Einsparung von Raum, Treibstoff und die Verringerung von $\mathrm{CO}_{2}$-Emissionen ermöglicht werden.

Das verwirklichbare Potenzial, welches Folienverpackung für die Reduktion an Lebensmittelabfällen birgt, hängt stark von der Kommunikation mit den KonsumentInnen und deren Informationsstand ab. Der Ruf von Folienverpackungen in der Öffentlichkeit muss ihrem Wert und ihrem Nutzen für Wirtschaft und Umwelt gleichermaßen gerecht werden. Hier ist es notwendig, geeignete Kampagnen für die Information der KonsumentInnen voranzutreiben, um es ihnen zu ermöglichen, informierte Entscheidungen bei ihrem Konsumverhalten zu treffen.

Während die Verpackung eines Produkes mittels Folien nur einen sehr geringen Teil seiner Umweltbelastung ausmacht, reduziert sich durch geeignet dimensionierte Verpackung die Masse an anfallendem Lebensmittelabfall, welcher, wie gezeigt, einen weitaus schädlicheren Einfluss auf die Umwelt hat. 
Um die positiven Vorzüge von Mono- und Multilayerverpackungen vollends auszunutzen, ist es notwendig, die EOL-Situation dieser Materialien $\mathrm{zu}$ erforschen und neue Technologien für deren Sammlung und Aufbereitung zu etablieren. Hierzu zählen einerseits entsprechende Sammelsysteme und andererseits neue Technologien, die, im besten Falle, bereits auf bestehenden Sortiertechnologien aufbauen und sich möglichst kostengünstig in bestehende Anlagen integrieren lassen.

Wie erwähnt ließe sich mit dem verstärkten Einsatz von Multilayer- und Monolayerverpackung eine Reduktion des Wasserverbrauchs um etwa $44 \%$ und eine $\mathrm{CO}_{2}$-Einsparung von etwa $40 \%$ realisieren. Nun ist Handeln ge- fragt, um dieses Gedankenexperiment Wirklichkeit werden zu lassen.

Funding Open access funding provided by Montanuniversität Leoben.

Open Access Dieser Artikel wird unter der Creative Commons Namensnennung 4.0 International Lizenz veröffentlicht, welche die Nutzung, Vervielfältigung, Bearbeitung, Verbreitung und Wiedergabe in jeglichem Medium und Format erlaubt, sofern Sie den/die ursprünglichen Autor(en) und die Quelle ordnungsgemäß nennen, einen Link zur Creative Commons Lizenz beifügen und angeben, ob Änderungen vorgenommen wurden.
Die in diesem Artikel enthaltenen Bilder und sonstiges Drittmaterial unterliegen ebenfalls der genannten Creative Commons Lizenz, sofern sich aus der Abbildungslegende nichts anderes ergibt. Sofern das betreffende Material nicht unter der genannten Creative Commons Lizenz steht und die betreffende Handlung nicht nach gesetzlichen Vorschriften erlaubt ist, ist für die oben aufgeführten Weiterverwendungen des Materials die Einwilligung des jeweiligen Rechteinhabers einzuholen.

Weitere Details zur Lizenz entnehmen Sie bitte der Lizenzinformation auf http://creativecommons.org/licenses/ by/4.0/deed.de.

\section{Literatur}

BMK (2021): Die Bestandsaufnahme der Abfallwirtschaft in Österreich - Statusbericht 2021. Statusbericht 2021 (Referenzjahr 2019). Bundesministerium für Klimaschutz, Umwelt, Energie, Mobilität, Innovation und Technologie. Available online at https://www.bmk.gv.at/dam/jcr: 04ca87f4-fd7f-4f16-81ec-57fca79354a0/BAWP Statusbericht2021, updated on 2021 [05.11.2021] Büsser, S. and Jungbluth, N. (2009): LCA of Chocolate Packed in Aluminium Foil Based Packaging

Büsser, S. and Jungbluth, N. (2011): LCA of Ready-to-Serve Goulash Soup Packed in StandUp Pouches.

Büsser, S., Steiner, R., Jungbluth, N. (2007): LCA of Food Products/Packed Goods: Coffee, butter, spinach.

Denkstatt (2016): How packaging contributes to food waste prevention. Aufgerufen: https:// www.save-food.org/cgi-bin/md_interpack/ lib/all/lob/return_download.cgi/3 Interpack 2017 denkstatt Packaging Food Waste Preven tion_V1.0.pdf?ticket=g_u_e_s_t\&bid=5684\&no mime_type $=0[05.11 .2021]$

Eco3E (2016): Materials-plastics. Aufgerufen http://eco3e.eu/base/plastics/ [05.11.2021]


in Austria: analysis of the status quo and environmental improvement potentials [Dissertation, Technische Universität Wien]. reposiTUm. https://resolver.obvsg.at/urn:nbn:at:at-ubtuw: 1- $115633[05.11 .2021]$

Flexible Packaging Association [FPA] (2009): Flexible packaging: contributing to sustainability. Aufgerufen: https://www.scribd.com/ document/275185809/Flexible-Packaging-
Contributing-to-Sustainability-Presentation [05.11.2021]

Ifeu (2014): Potential packaging waste prevention by the usage of flexible packaging and its consequences for the environment.

Morris, B. A. (2016): The Science and Technology of Flexible Packaging: Multilayer Films from Resin and Process to End Use. Saint Louis: Elsevier Science, ISBN: 9780323243254

Pira (2011): The future of Global Packaging Market forecasts to 2010. Aufgerufen: https:// www.yumpu.com/en/document/read/5114572/ market-statistics-and-future-trends-in-globalpackaging [05.11.2021]

PlasticsEurope (2015): Plastics-the Facts 2015 An analysis of European plastics production, demand and waste data.

Scharff, C. (2021): Was tun bei Kreislaufproblemen? KC-Fachtagung Recycling Days 2021 7.Juni-8. Juni 2021. Online, 7.Juni.

Sealed Air (2014): Grocery Shoppers and Food Waste in the United States.

Silvenius, F., Katajajuuri, J. M., Grönman, K., Soukka, R., Koivupuro, H. K., \& Virtanen, Y. (2011): Role of packaging in LCA of food products. In Towards Life Cycle Sustainability Management (pp. 359-370). Springer Netherlands. Steinert (2018) UniSort BlackEyeWorld News: erstes wirtschaftlich arbeitendes optisches Sortiersystem zur Erkennung und Sortierung von schwarzen Kunststoffen. https://steinertglobal. $\mathrm{com} /$ fileadmin/user_upload/_steinert/

downloads/_magnete-sensorsortierer/

_sensorsortierung/UniSort_BlackEye/STE

UNI_blackeye_DE.pdf. Aufgerufen am 05.11.2021.
The Consumer Goods Forum (2011): A Global Language for Packaging and Sustainability. A framework and measurement system for our industry

The European Parliament and the Council of the European Union (2018): Directive (EU) 2018/851 of the European Parliament and of the Council of 30 May 2018 amending Directive 2008/98/EC on waste („EU Waste Framework Directive").

Walker, T. W., Frelka, N., Shen, Z., Chew, A. K., Banick, J., Grey, S., Kim, M. S., Dumesic, J. A., Van Lehn, R. C., Huber, G. W. (2020): Recycling of multilayer plastic packaging materials by solvent-targeted recovery and precipitation. Sci. Adv. 6, eaba7599. https://doi.org/10.1126/ sciadvaba7599

Wohner, B., Pauer, E., Heinrich, V., Tacker, M. (2019): Packaging-Related Food Losses and Waste: An Overview of Drivers and Issues. Sustainability, 11 (1):264.

Woidasky, J, Lang-Koetz, C. Heyde, M., Wiethoff, S., Sander, I., Schau, A., Moesslein, J., Fahr, M., Richards, B., Turshatov, A., Sorg, F. (2018) Tracer Based Sorting-Innovative Sorting Options for Post Consumer Products.

Hinweis des Verlags Der Verlag bleibt in Hinblick auf geografische Zuordnungen und Gebietsbezeichnungen in veröffentlichten Karten und Institutsadressen neutral. 